\title{
Editorial for the special issue of service computing and service oriented enterprise systems in design, manufacturing and supply chain
}

\author{
Ying Liu • Rossi Setchi • Vincent S. Tseng • \\ Feng Chun Wang
}

Received: 20 March 2012 / Accepted: 24 March 2012 / Published online: 8 April 2012

(C) Springer Science+Business Media, LLC 2012

It has been witnessed that the world is gradually evolving into a service based economy with a notable figure that around $70 \%$ of workforce is now working in the service sector in the US (2007). In response, the global industry is moving very fast to embrace the merits offered by service science. In the context of design, manufacturing and supply chain, service-oriented computing and its corresponding enterprise system offer solutions towards various challenging issues of global design collaboration, manufacturing resource allocation and service delivery. Service-oriented architecture and systems have been proposed to support the integration and collaboration of distributed resources, where a service can be ubiquitously requested to process certain tasks or combined with other services to devise a customized functionality. A variety of forward-looking research and applications have emerged, including for example, service oriented design coordination, service oriented Semantic Web service for business integration, service driven collaborative manufacturing execution platform, offshore manufacturing and supply chain management based on SOA, etc. With the emergence of these interesting research areas, this Special Issue is dedicated to the latest advances, innovations, state-ofthe-art research, technology development and applications of

\author{
Y. Liu ( $\varangle)$ \\ Department of Mechanical Engineering, National University of \\ Singapore, Singapore 117576, Singapore \\ e-mail: mpeliuy@nus.edu.sg \\ R. Setchi \\ Cardiff University, Cardiff, UK \\ V. S. Tseng \\ National Cheng Kung University, Tainan, Taiwan \\ F. C. Wang \\ IBM China Research Lab, Shangdi, China
}

service computing and service oriented enterprise systems in the context of design, manufacturing and supply chain.

The first paper, "Service-Oriented Architecture for Ontologies Supporting Multi-Agent System Negotiations in Virtual Enterprise" by Wang, Wong, and Wang, considers the coordination of agents, as virtual enterprise delegates, under heterogeneous enterprise knowledge representation and service oriented architecture (SOA). Ontology, as a basis for enterprise knowledge representation and negotiation, is adopted to research semantic interoperability for realizing multi-agent negotiations. Their proposal of property matching methods, a correspondence-based extended contract net protocol and a fuzzy set theory based knowledge reuse method, have presented a practical approach for coordinating and enabling services in the virtual enterprise life cycle. The approach indicates that semantic ambiguity during agent based communication that arises due to heterogeneous knowledge modeling can be resolved using ontology. In light of the promising results achieved, issues such as security and privacy under service-oriented multi-agent communications can be further investigated.

In line with one of the special issue topics on enterprise service security, trust and privacy, the second paper, "Mitigating the Risk of Information Leakage in a Two-Level Supply Chain through Optimal Supplier Selection" by Zhang, Cao, Wang and Zeng focuses on the evaluation and mitigation of risk associated with information leakage in a two-level supply chain network. Their research can be divided in two main stages: Firstly, the problem of information leakage in supply chain that considers potential competition between supplier and manufacturer is formulated. Secondly, the risk associated with information leakage is mitigated through optimal supplier selection. Based on the probabilities of information leakage calculated, the authors have demonstrated the feasibility of their approach via an optimal regeneration of system 
supplier allocation where the potential risks of information leakage can be feasibly mitigated.

The remaining papers included in this special issue can be clustered into two primary groups. The first group emphasizes on service-oriented process evaluation, selection and management. "An Integrated Modeling Method for Assessment of Quality Systems Applied to Aerospace Manufacturing Supply Chains" by Harun and Cheng proposes an assessment approach for business process re-engineering through a systematic integrated modeling. In particular, they aimed to study the impacts towards existing engineering process due to the introduction of new standards. Based on the existing IDEF0 standard, an integrated modeling approach, IDEF-Q, is proposed for process representation and assessment. Their approach is successfully exemplified through a manufacturing supply chain for an aerospace company, where the conformity of the process model under new standards and its corresponding areas for improvement can be intuitively identified.

"Service Process Simulation for Integrated Service Evaluation" by Watanabe, Mikoshiba, Tateyama and Shinomura describes their efforts in representing and evaluating service process through a simulation model. A service evaluation framework, named integrated service evaluation framework, is proposed to assess the influence of a service process from multiple stakeholders' viewpoints. Modeling wise, scene transition net (STN) is proposed as a simulation model structured on IDEF0 based service process model. Their proposal is verified using a case study of bicycle rental service. The results indicate that crucial model outputs such as average travel time and revenue can be feasibly determined under different usage scenarios of bicycle availability. We reckon that their approach can also be extended to enterprise service modeling and evaluation.

"VCS: Value Chains Simulator, a Tool for Value Analysis of Manufacturing Enterprise Processes (A Value-Based Decision Support Tool)" by Bosch-Mauchand, Siadat, Perry and Bernard works on modeling, simulation and decision making on manufacturing enterprise processes using a value estimation approach. Value chain simulator is suggested as a discrete event simulator tool for value evaluation related to process aspects, such as balancing cost, quality and delivery time. Analytical hierarchy process is embedded as one of their core analytical methods. For validation, a case study in the microelectronics field is conducted to simulate its decision making process on the choice of manufacturing resource. Given its global product value chain indicators, the best scenario that benefits all stakeholders is selected. Further improvement can be leveraged via investigating a suitable search strategy through optimization.

"Evaluation Models for Service Oriented Process in Spare Parts Management" by Cheng and Prabhu analyzes service-oriented process from the perspective of technology complexity and system performance using two analysis models. The first model studies complexity issue which is expressed as a function of inter-activity and intra-activity complexity. Secondly, based on a queuing network model, performance model analyzes service utilization, throughput, response time and queue length to estimate service duration time. A case study on tool replenishment processes is used to compare between manual process and RFID-based automated process. Their results reveal that RFID-based process has higher usability and accuracy with less procurement complexity when compared to manual process. We value the practical benefits of their proposal for rapid comparison among different process solutions in designing a large scale, complex service-oriented process under changing process requirements.

The second group reports current studies on the product design perspective, where design assessment process that involves design change and imprecise customer inputs can be further enhanced. "A Network-based Assessment Approach for Change Impacts on Complex Product" by Cheng and Chu explores a quantitative method to systematically

evaluate the potential change impacts caused by a complex product. Based on the theory of weighted networks, the connections of different parts in a complex product are first identified and modeled to form a product network. In order to perform change analysis in an objective manner, three changeability indices are suggested for change impact assessment. Using a graph-based search algorithm, the authors have demonstrated the merits of their approach through a case study of root blowers where change propagation can be feasibly identified. We are certainly interested in seeing further applications of this approach for designing complex products or systems.

"An Optimum Design Selection Approach for Product Customization Development" by Liu, Ramirez-Serrano and Yin attempts to tackle product customization evaluation and decision making based on the inputs from customers and designers which are often uncertain. A fuzzy-based decision making strategy is adopted, where fuzzy sets represent the imprecise demands of customers and designers and fuzzy analysis investigates customers' responses to various design options. Multi-criteria customer utility is identified through conjoint analysis. A multi-attribute analysis diagram is then developed to visualize the preference of each attribute from the experts' group decision. Their case study on mobile robot selection indicates that the proposed approach is able to assist engineers in evaluating design alternatives especially when they are subject to different qualitative design and customer criteria.

The eight articles selected for this Special Issue were chosen after two rounds of reviews, from 16 initial intentions. In the first round, each paper was peer reviewed by three 
reviewers, and in the second round, the revised articles were reviewed by one former reviewer and two Guest Editors. A third revision was requested for a small number of articles, more for language editing. Finally, we would like to take this opportunity to thank all authors for their excellent work contributing to this Special Issue. We are very grateful to the reviewers for offering their precious time and effort and for providing constructive comments. Particularly, we would like to express our sincere thanks to Prof. Andrew Kusiak,Editor-in-Chief, and Prof. Roger Jiao, Associate Editor, for giving us the opportunity to make this Special Issue a reality. Finally, we also thank Anitha Sankar from Springer for her editorial assistance. Without all of you, this Special Issue would not be possible. 